\title{
An Efficient Design Procedure to Implement the Fractional-Order Chaotic Jerk Systems with the Programmable Analog Platform
}

\author{
Nimet Korkmaz ${ }^{\mathbb{D}} *, 1$ and Ibrahim Ethem SACU ${ }^{1 D} \alpha, 2$ \\ ${ }^{*}$ Department of Electrical and Electronics Engineering, Kayseri University, Kayseri 38280, Turkey, ${ }^{\alpha}$ Department of Clinical Engineering Research Center, \\ Erciyes University, Kayseri 38039, Turkey.
}

ABSTRACT An effective design procedure has been introduced for implementing the fractional order integrator structures with a modified low pass filters (LPFs) and its functionality is verified by realizing a fractional-order chaotic system. In these applications, the state variables of the fractional-order Sprott's Jerk system are emulated by these first order LPFs. Since the discrete device based designs have the hard adjustment features and the circuit complexities; the realizations of these LPFs are carried out with the Field Programmable Analog Arrays (FPAAs), sensitively. Hence, the introduced LPF based method has been applied to the fractional order Sprott's Jerk systems and these fractional-order systems, which are built by the several nonlinear functions, have been implemented with a programmable analog device. In this context, the minimum fractional-orders of the Sprott's Jerk systems are calculated by considering the stability of the fractional-order nonlinear systems. After that, these systems are simulated by employing the Grünwald-Letnikov (G-L) fractional derivative method by using a common fractional-order. Thus, the stability analyses of the fractional-order Sprott's Jerk system are supported by the numerical simulation results. After the numerical simulation stage, the design procedures of the FPAA based implementations of the Sprott's Jerk systems have been dealt with in detail. Finally, thanks to the introduced first-order LPF method, the hardware realizations of the Sprott's Jerk systems have been achieved successfully with a single FPAA device.

\section{KEYWORDS}

Fractional-order nonlinear system Sprott's Jerk System Low pass filter FPAAs

\section{INTRODUCTION}

After the discovery of the Lorenz's chaotic system, the chaos concepts, the chaotic stability and the circuit implementations of the chaotic structures are well documented in the literature. Simulations of the chaotic attractors by solving two- or higher dimensional ordinary differential equations with numerical analysis tools or generations of these structures by emulating their mathematical descriptions with the several electronic hardware have become more interesting in the last few decades (Kiliç 2010). However, expressing a nonlinear system with simple definitions is very important in terms of both adaptation of the system to different

Manuscript received: 14 July 2021 ,

Revised: 9 August 2021,

Accepted: 11 August 2021.

${ }^{1}$ nimetkorkmaz@kayseri.edu.tr (Corresponding Author)

2 iesacu@erciyes.edu.tr research fields and applicability to the problems in real life. Thus, these systems have been studied easily; also they have found an extensive application field in scientific engineering problems such as quantum chaos (Stöckmann 1999), chaos-based secure communications (Chen and Ueta 2002), to truly random number generator (Öztürk and Kılıç 2019) and the fractional-order definitions of the chaotic systems (Tlelo-Cuautle et al. 2020). Among these scientific accretions about the chaotic systems, Sprott's chaotic models have taken a considerable attention in the literature thanks to their simplicities and rich contents (Sprott 1994). This simple chaotic system is based on "Jerk systems" and the source of its rich contents is to include the different nonlinear functions in the system definition (Sprott 1997, 2000b,a; Ahmad and Sprott 2003).

The simplicities of the nonlinear systems provide an extra advantage for the fractional-order definition, because the fractional order models have the extra degrees and these methods enrich the analysis with more details in new dimensions. Thus, the real time 
problems and systems are able to be modeled by the fractional equations more accurately than the integer ones. Fractional calculus has found various applications in areas such as control system theory, biochemistry and medicine, circuit theory and design (Elwakil 2010). While the fractional-order PID controllers provide an extra freedom for tuning of time and frequency responses in the control system theory (Deniz et al. 2019); the fractional calculus is used for modeling the measured impedance versus the frequency of the investigated material in the biochemistry (Azar et al. 2018). Besides, the fractional capacitor (-fractance) or fractional inductance concepts are brought in the literature and these structures are adapted to the canonical theories of the integer order RC-RL-RLC circuits in the circuit theory (Krishna and Reddy 2008; Atangana and Alkahtani 2015). The main circuit applications such as oscillators, filters, differentiators and integrators etc. are also built by utilizing their fractional-order definitions (Radwan et al. 2008; Sacu and Alci 2019; Chen and Moore 2002; Charef 2006). The most important advantages of the fractional-order systems are able to be listed as follows: i) The high frequency real-time signals are generated with the fractional-order oscillators (Ahmad et al. 2001), ii) Both the frequency and the phase shifts among these generated signals are controlled by tuning the fractional-orders of the oscillators (Maundy et al. 2012), iii) The fractional-order differentiators and integrators provide adjustable phase shifts depending on the fractional order (Krishna 2011), iv) The slope of the filter response, the cutoff frequency (-center frequency) and the quality factor in the fractional filters are changed by adjusting the fractional-orders (Radwan et al. 2009), and v) the fractional-order controllers can provide robust performance (Caponetto 2010). Considering the advantages listed above, the fractional-order chaotic oscillators have become the one of the most important research fields of the nonlinear circuits and systems in the resent years.

The usage of the numerical simulation results is an often referred approach to demonstrate the effectiveness of the obtained results in the scientific studies, which are about the fractional-order chaotic oscillators. Two alternative numerical analyses methods are commonly used to simulate the fractional-order chaotic oscillators (Arena 2000; Chen et al. 2016). First of them is based on Grünwald-Letnikov (G-L) fractional derivative that is used for calculation of the time domain responses of the studied systems and this method demands more memory size. In the second method, the fractional integral operator $1 / s^{q}(0<\mathrm{q}<1$ is fractional order $)$ is approximated by the high order integer transfer functions within a limited frequency band. These high order integer transfer functions are generally implemented by the R-C networks, but there is a tradeoff between simplicity of the implementation and frequency band in this method. On the other hand, although the circuit implementations of the fractional-order chaotic systems have impressive advantages, the number of their hardware implementations is limited. A large part of these studies are usually realized with discrete devices by using the coupled R-C circuits (Radwan and Salama 2012; Gómez et al. 2013; Gómez-Aguilar et al. 2017). Also, since the programmable and reconfigurable analog/digital devices have several effective specialties such as the flexible designing, the low time and equipment costs and the rapid prototyping, a few the programmable analog/digital devices based hardware validation studies about the fractional-order chaotic systems are also available in the literature (Petráš 2011; Singh et al. 2020). However, the followed processes in these available studies do not offer a common design procedure in order to implement programmable analog device based implementations of the fractional-order chaotic systems.
In this context, an effective alternative design process will be suggested for realizing of the fractional-order chaotic systems with the electronic hardware in this study. In this introduced procedure, the state variables of the fractional-order chaotic systems are emulated by the modified first order low-pass filters (LPFs), so the hardware usage cost and the circuit complexities have been decreased at the beginning of the hardware design process. The analog filter designs and their realizations are carried out with the Field Programmable Analog Arrays (FPAAs), logically. Thus, the introduced method has been applied to the fractional order Sprott's Jerk systems and the best of our knowledge, the fractional order Sprott's Jerk systems, which are built by the several nonlinear functions, have been implemented with a programmable analog device. To this end, the minimum fractional-orders of the state variables of the Sprott's Jerk systems are derived by taking into stability of the nonlinear systems consideration at the equilibrium points. After the determination of the fractional orders, the Sprott's Jerk systems, which include several nonlinear functions, are simulated by employing the G-L fractional derivative method. Then, the details of the introduced alternative design process have been handled. Finally, by means of this method, the hardware realizations of the Sprott's Jerk systems have been achieved successfully with a single FPAA device.

This paper is organized as follows: The general background about the Sprott's Jerk systems and their fractional-order counterparts are given in Section 2. The main definition of the G-L fractional derivative method and the numerical simulation results of the fractional-order Sprott's Jerk systems are also presented in Section 2. The alternative design procedure is introduced in Section 3 and the FPAA based implementation results of the fractional-order Sprott's Jerk systems are also given in this section. The discussions about the performance evaluations of the introduced methods and the concluding remarks are given in the last section.

\section{THE GENERAL BACKGROUND ABOUT THE FRACTIONAL- ORDER SPROTT'S JERK SYSTEMS}

In 1994, Sprott offered several simple chaotic systems (Sprott 1994). After the response of the Gottlieb's question about the Jerk function (Gottlieb 1996), these simple chaotic systems have been adapted to an explicit third order form as $\dddot{x}=J(x, \dot{x}, \ddot{x})$. These redefined systems have been called Sprott's Jerk systems and defined by to following equations (Sprott 1997, 2000b,a; Ahmad and Sprott 2003):

$$
\begin{aligned}
& \frac{d x}{d t}=\dot{x}=y \\
& \frac{d^{2} x}{d t}=\ddot{x}=\dot{y}=z \\
& \frac{d^{3} x}{d t}=\dddot{x}=\ddot{y}=\dot{z}=F(x)-p z-y
\end{aligned}
$$

where, ' $x$ ', ' $y$ ' and ' $z$ ' are the state variables of this system. ' $F(x)$ ' is a nonlinear function and plays an important role in the chaos mechanism of the system. Several chaotic structures with different characteristics have been obtained by using these piecewise linear functions (PWLs), which have different nonlinear definitions as seen in Table 1 (Sprott 1997, 2000b,a; Ahmad and Sprott 2003). ' $p$ ' is a control parameter, the chaotic dynamics of this system change by depending on the values of ' $p$ '. 
Table 1 The nonlinear functions of the Sprott's Jerk system and the values of the nonlinear function's parameters

\begin{tabular}{llll}
\hline The Nonlinear Functions-' $F(x)$ & Eq. No & Function Parameters & The minimum fractional orders \\
\hline$F_{1}(x)=|x|-r$ & $(2)$ & $r=2, p=0.35$ & $q_{\min }>0.885$ \\
$F_{2}(x)=-B x+C \operatorname{sgn}(x)$ & $(3)$ & $B=1.2, C=2, p=0.6$ & $q_{\min }>0.904$ \\
$F_{3}(x)=B\left(x^{2} / C-C\right)$ & $(4)$ & $B=0.58, C=1, p=0.42$ & $q_{\min }>0.831$ \\
$F_{4}(x)=B x\left(x^{2} / C-1\right)$ & $(5)$ & $B=1.6, C=5, p=0.4$ & $q_{\min }>0.848$ \\
$F_{5}(x)=-B x\left(x^{2} / C-1\right)$ & $(6)$ & $B=0.9, C=0.47, p=0.4$ & $q_{\min }>0.837$ \\
$F_{6}(x)=-B[x-2 \tanh (C x) / C]$ & $(7)$ & $B=2.15, C=1, p=0.58$ & $q_{\min }>0.864$ \\
\hline
\end{tabular}

In the literature, several chaotic oscillators such as Chua, Rössler, Duffing etc. have been redefined by using the fractionalorder integrators (Petráš 2011). The Sprott's Jerk system has been also transported a fractional order system in Ref (Ahmad and Sprott 2003). In Ref (Ahmad and Sprott 2003), only one state variable (' $x$ ') is given as a fractional-order integrator. In our study, all state variables are dealt with as the fractional-order integrators and their fractional-orders are set to equal values. The values of the fractional-order are calculated by considering the stability of these nonlinear systems.

The stabilities of the fractional order nonlinear systems are as important as in the integer-order nonlinear systems. Although the Lyapunov criterion is commonly employed for the stability of the integer nonlinear systems, this criterion is not valid for the fractional-order nonlinear systems. There are two methods in order to check the stabilities of the fractional-order nonlinear systems: In the case of the commensurate order fractional system, namely $q_{1}=q_{2}=\ldots . .=q_{n}=q$, Tavazoei and Haeri (Tavazoei and Haeri 2007) have proposed a method. According to this method, if the arguments of all the eigenvalues $\left[\lambda_{i}(i=1,2, \ldots . ., n)\right]$ of the system satisfy the $\left|\arg \left(\lambda_{i}\right)\right|>\frac{q \pi}{2}$ condition, the equilibrium points of this system are asymptotically stable. On the other hand, in the case of the incommensurate order fractional system, namely $q_{1} \neq q_{2} \neq$ $\ldots . \neq \neq q_{n} \neq q$, the stability of the system is determined as following (Tavazoei and Haeri 2008): If the arguments of all the roots $\lambda$ of the Eq.8 satisfy the $\left|\arg \left(\lambda_{i}\right)\right|>\frac{\pi}{2 m}$ condition, this system is the asymptotically stable, where $\mathrm{m}$ is the least common factor of the denominators of fractional orders and ' $J$ ' is the Jacobian matrix.

$$
\operatorname{det}\left(\operatorname{diag}\left(\left[\begin{array}{llll}
\lambda^{m q} & \lambda^{m q} & \ldots & \lambda^{m q}
\end{array}\right]\right)-J=0\right.
$$

As mentioned before, all fractional-orders of the Sprott's Jerk systems are set to equal values in order to get a simple stability analysis and the fractional-order Sprott's Jerk system is given as in Eq. 9.

$$
\begin{aligned}
& \frac{d x^{q}}{d t^{q}}=y \\
& \frac{d y^{q}}{d t^{q}}=z \\
& \frac{d z^{q}}{d t^{q}}=F(x)-p z-y
\end{aligned}
$$

The fractional-order of the Sprott's Jerk systems including a PWL function in Eq.3 has been calculated as in the following part in terms of being an example of the stability analysis of the fractional-order nonlinear systems. Firstly, the equilibrium points of the system are derived by equaling the right hand side of the equations in Eq.9 to zero. Then, three of equilibrium points of Eq. (9) are calculated for $p=0.6$ and the values of the equilibrium points are reported as $E_{1}(-2 / 1.2,0,0), E_{2}(0,0,0)$ and $E_{1}(2 / 1.2,0,0)$. The eigenvalues of this system are calculated as $\left({ }^{\prime} 0.1619+\mathrm{i} 1.1282^{\prime},{ }^{\prime} 0.1619-\mathrm{i} 1.1282^{\prime},{ }^{\prime}-0.9237^{\prime}\right)$ by utilizing the values of the equilibrium points with the $\operatorname{det}(\lambda I-J)=0$ formula. After the determinations of the eigenvalues, the minimum fractional order is identified by using the method proposed by Tavazoei and Haeri (Tavazoei and Haeri 2007). The minimum fractional orders for the Sprott's Jerk system including the nonlinear functions in Eqs.2-7 are calculated by following these procedures and their values are also reported to Table 1.

After these calculations, the fractional-orders of the Sprott's Jerk systems are identified as $q=0.95$ in order to provide a robust stability for all applications in this study. Then, the nonlinear functions in Table 1 have been adapted to the Eq.9 and the employability of the fractional-order has been verified by observing the results of the numerical analyses. Several effective methods such as the Riemann-Liouville, Caputo and Grünwald-Letnikov are improved for calculating the fractional derivatives in the literature (Arena 2000; Oldham and Spanier 1974; 199 1999). Here, the G-L method has been preferred due to its prevalent usage in the numerical analysis of the chaotic systems. This method is used for the numerical analyses of the Sprott's Jerk systems and this method is defined as in Eq. 10.

$$
{ }_{a} D_{t}^{q} f(t)=\lim _{h \rightarrow 0} \frac{1}{h^{q}} \sum_{j=0}^{\left[\frac{t-a}{h}\right]}(-1)^{j}\left(\begin{array}{l}
q \\
j
\end{array}\right) f(t-j h)
$$

Where ' $a$ ' and ' $t$ ' are bounds of derivative operation, ' $h$ ' is the time step, ' $\left[\frac{t-a}{h}\right]$ ' means the integer part of the function and the binomial coefficients are expressed in terms of Gamma ' $\Gamma(*)^{\prime}$ ' function as in Eq.11.

$$
\left(\begin{array}{l}
q \\
j
\end{array}\right)=\frac{\Gamma(q+1)}{\Gamma(j+1) \Gamma(q-j+1)}
$$

Here, the G-L numerical analyses method has been applied to the fractional-order Sprott's Jerk systems, successfully. The obtained numerical simulation results of these systems are given in Figs.1a-f for the nonlinear functions in Eqs.2-7, respectively. 
The value of the ' $j$ ' parameter is set to 5000 and ' $h$ ' is equal to 0.1 . The initial conditions of the state variables are adjusted as $[(\mathrm{x}(0)=0.1, \mathrm{y}(0)=0, \mathrm{z}(0)=0]$ in all numerical simulations.

As seen from the numerical simulation results given in Fig.1, the fractional-order Sprott's Jerk systems exhibit the chaotic behaviors for the $q=0.95$ value. Additionally, these results support the results of the stability analyses in the previous part. Therefore, the all fractional orders of the Sprott's Jerk systems are taken as 0.95 in the following parts.
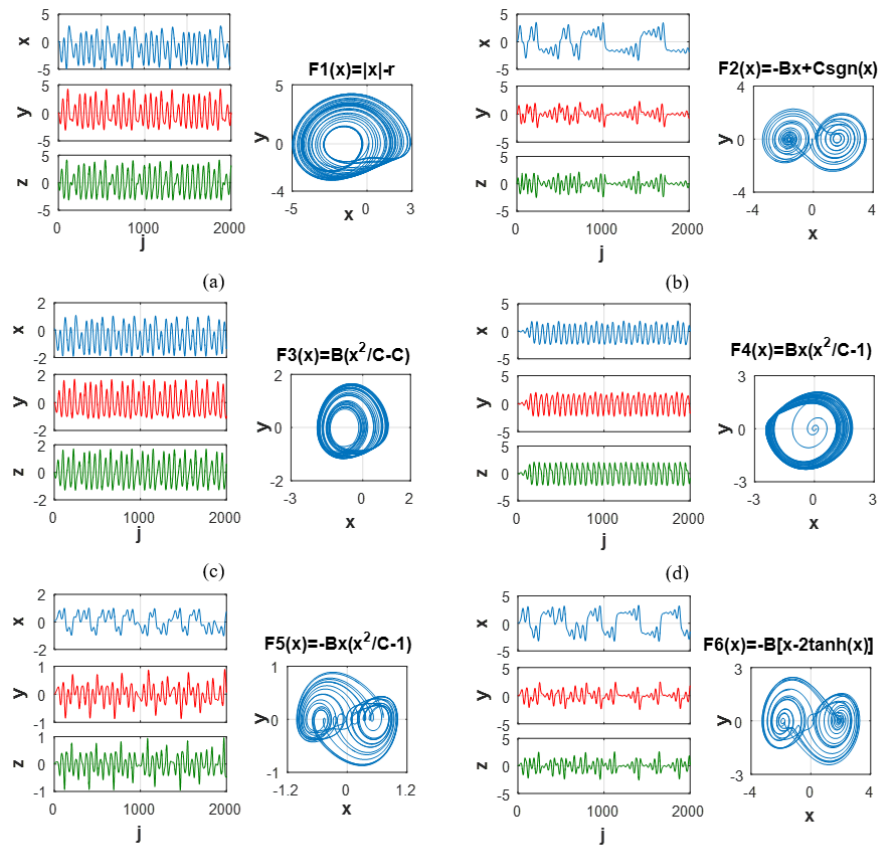

(e)

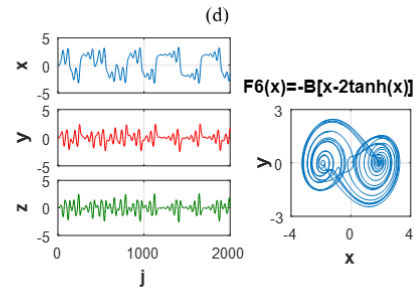

(f)

Figure 1 The numerical simulation results of the fractional-order Sprott's Jerk systems including the a) $F_{1}(x)$ function in Eq.2, b) $F_{2}(x)$ function in Eq.3, c) $F_{3}(x)$ function in Eq.4, d) $F_{4}(x)$ function in Eq.5, e) $F_{5}(x)$ function in Eq.6, and f) $F_{6}(x)$ function in Eq.7

\section{THE APPLICATION OF THE PROPOSED METHOD TO THE SPROTT'S JERK SYSTEMS BY UTILIZING THE FPAA DE- VICE}

Although the time domain responses of the fractional-order nonlinear systems are observed with the G-L method in the numerical simulation studies, it is very hard to realize this method with the electronic hardware because of the requirement to storage of the previous calculations. Thus, the high order transfer function approximations of the fractional integration operator $1 / s^{q}$ are able to be qualified as an alternative analyzing method for the hardware realizations of the fractional-order nonlinear systems. In the literature, these high order transfer functions are widely derived from the systematic methods such as the continued fraction expansion (CFE), Carlson, Oustaloup, Matsuda and Valsa (Khovanskii 1963; Carlson and Halijak 1964; Oustaloup et al. 2000; Matsuda and Fujii 1993; Valsa et al. 2011). These high order approximation transfer functions are framed in a limited frequency band and they are implemented with the combinations of the R-C, R-L or R-L-C pairs. However, it is preferred to use of the R-C networks for their implementation easiness, and there is also a tradeoff between simplicity of the implementation and frequency band in this method.
Additionally, as the order of the approximation function increases, the count of the employed passive components increases also, so an alternative method is introduced to overcome these problems in this study. In this proposed method, the fractional integrator operator is considered as a single parallel connected R-C pair in a limited frequency band with an acceptable error. This connection corresponds to a first order low pass filter (LPF) and the transfer function of this LPF is defined by the following equation:

$$
H(s)=\frac{V_{\text {out }}(s)}{V_{\text {in }}(s)}=\frac{k_{1}}{s+k_{2}}
$$

In Eq12, while ' $k$ ' is the zero of the LPF and its value is calculated by using the formula in Eq.13, ' $k_{2}$ ' is the pole of this LPF and its value is identified by the formula in Eq.14.

$$
\begin{gathered}
k_{1}=w_{\max }{ }^{1-q} / \sin \left(\frac{q \pi}{2}\right) \\
k_{2}=w_{\max } / \tan \left(\frac{q \pi}{2}\right)
\end{gathered}
$$

where while ' $q$ ' is the fractional order of the nonlinear system, ' $w_{\text {max }}$ ' is the radial frequency of the nonlinear system. The frequency response of a fractional-order integrator is shown in the bode diagram in Fig.2. In this figure, the black line represents an ideal fractional-order integrator for $q=0.95$, the blue dotted line is plotted for the proposed LPF in Eq.12. The ' $q$ ' and ' $w_{\text {max }}$ ' parameters of this LPF are set to 0.95 and $100 \mathrm{rad} / \mathrm{s}$, respectively. The value of the radial frequency is adjusted to this value arbitrarily similar to Ref (Ahmad and Sprott 2003). However, the proposed $\mathrm{LPF}$ closes to the ideal frequency response at this value.

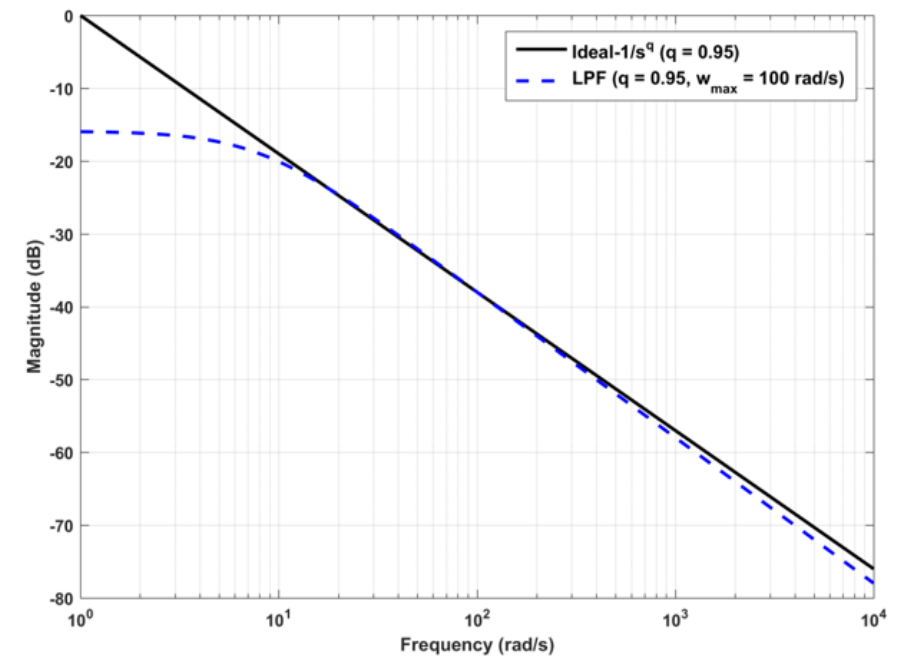

Figure 2 The bode diagram of an ideal fractional order system for $q=0.95$ and the proposed LPF $\left(q=0.95\right.$ and $w_{\max }=100$ $\mathrm{rad} / \mathrm{s})$

As mentioned in the introduction part, the circuit implementations of the fractional-order chaotic systems have impressive advantages, but the number of their hardware implementations is limited. Thus, here, an effective alternative design process has been suggested for realizing of the fractional-order chaotic systems with the electronic hardware in an easy way. In this introduced procedure, the LPF's characteristic in Eq.12 is adapted to the fractional-order nonlinear systems properly and this modified LPF structure can be used successfully instead of the fractionalorder integrators in the in Eq.9. Thus, the hardware usage cost and 
the circuit complexities have been decreased at the beginning of the hardware design process. To this end, after the Sprott's Jerk system is transported to the s-domain (the initial conditions are accepted as zero), its definition can be rewritten as in the following for adapting to the proposed LPF's characteristic:

$$
\begin{aligned}
& X(s)=\left[\left(Y(s)+k_{2} X(s)\right)\left(1 / k_{1}\right)\right]\left[\frac{k_{1}}{\left(s+k_{2}\right)}\right] \\
& Y(s)=\left[\left(Z(s)+k_{2} Y(s)\right)\left(1 / k_{1}\right)\right]\left[\frac{k_{1}}{\left(s+k_{2}\right)}\right] \\
& \left.Z(s)=\left[(F(s)-p Z(s)-Y(s))+k_{2} Z(s)\right)\left(1 / k_{1}\right)\right]\left[\frac{k_{1}}{\left(s+k_{2}\right)}\right]
\end{aligned}
$$

After these arrangements, a representative illustration of the obtained configuration is given in Fig. 3. In this figure, the LPFs should provide the characteristic in Eq. 12. Although the implementation issue of the fractional-order nonlinear system is solved with this design, the adjustment of the R-C pairs' values is hard to get the characteristic in Eq.12. Thus, the analog low pass filter designs and their realizations are carried out with the Field Programmable Analog Arrays (FPAAs) very sensitively by using the "BILINEAR FILTER" block in this device.

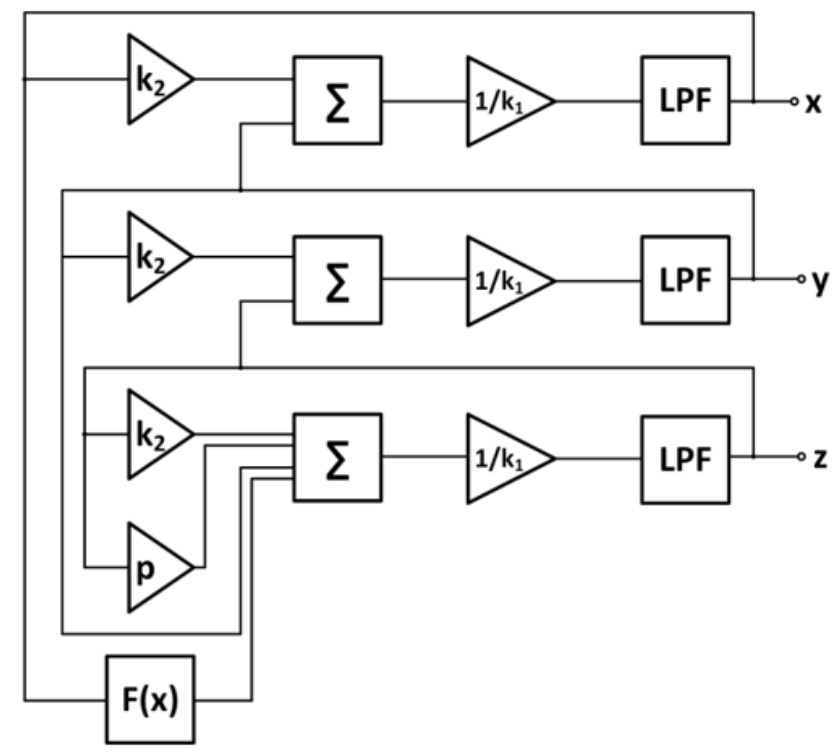

Figure 3 A representative illustration of the LPF in Eq. 12 based design of the fractional-order Sprott's Jerk system

The FPAA device offers the flexible designing, the low time and equipment costs, programmability and reconfigurability properties and the rapid prototyping features by programming a matrix of the elements. These programmable elements are called Configurable Analog Blocks (CABs). The Configurable Analog Modules (CAMs) in the FPAA device are built by using the switched-capacitor technology, so the predefined CAM block in the FPAA can be programmed easily in order to get the desired configurations. The list of these CAM blocks and their functions are available in the ANADIGM DESIGNER ${ }^{T M}$ tool. According to this tool the "BILIN-

EAR FILTER" CAM block ("N-") has the characteristic as in Eq. 16:

$$
\operatorname{LPF}(s)=\frac{V_{\text {out }}(s)}{V_{\text {in }}(s)}= \pm \frac{2 \pi f_{0} G}{s+2 \pi f_{0}}
$$

where, ' $G$ ' is the adjustable gain value and ' $f_{0}$ ' is the corner frequency of this LPF. The characteristic of the "BILINEAR FILTER" CAM block in Eq.16 is very similar to the LPF in Eq.12 that is proposed to realize the fractional-order integrators. Therefore, the "BILINEAR FILTER" block can be used to implement the desired LPF in the FPAA based realizations of the Sprott's Jerk systems. The remained mathematical descriptions in Eq.15 are also realized by using the "SUM DIFF" (" ) and the "TRANSFER FUNCTION" ( " $)$ blocks. An example of the FPAA based design scheme of the Sprott's Jerk system is given in Fig.4. In this figure, while the "SUM DIFF" block creates a half cycle summing/ subtraction stage with up to four inputs, the "TRANSFER FUNCTION" block implements a user specified voltage transfer function with 256 quantization steps. The nonlinear functions in Table 1 have been embedded to the "TRANSFER FUNCTION" blocks in all designs. On the other hand, the FPAA device has a saturation level $( \pm 2 \mathrm{~V})$, so the studied model must be rescaled according to this saturation level. After these modifications, the coefficients of the Sprott's Jerk systems in Eq.15 have been rearranged and their final values are given in Table 2. The gain inputs of the "SUM DIFF" blocks have been set to these values. Additionally, the ' $k_{1}$ ' and ' $k_{2}$ ' parameters of the LPF are calculated as 1.2628 and 7.8702 respectively for $q=0.95$ and $w_{\max }=100 \mathrm{rad} / \mathrm{s}$.

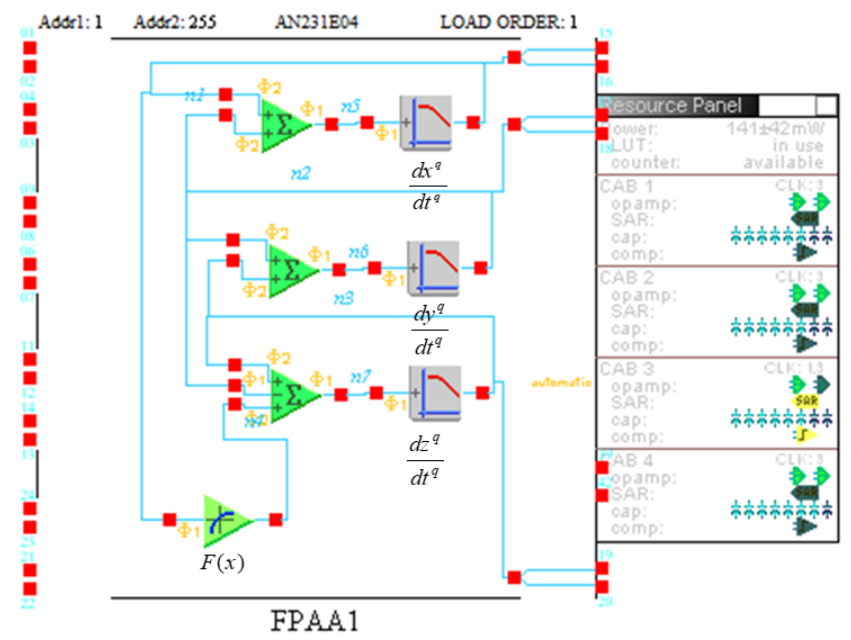

Figure 4 An example FPAA design scheme of the fractionalorder Sprott's Jerk system

After the similar configurations are completed in ANADIGM DESIGNER $^{T M}$ tool for all nonlinear functions in Table 1, the designed models are downloaded to the AN231E04 FPAA board one by one via a serial interface by utilizing the FPAA board's programmability and reconfigurability properties. The less power consumptions and the CAB usages are also seen in Fig. 4 and they are common in all realizations for the different nonlinear function based Sprott's Jerk system. Additionally, only a single FPAA device has been used in these realizations in contrast to the available FPAA based applications of the fractional-order chaotic systems in the literature (Petráš 2011). A photograph of the experimental setup is seen in Fig.5 and the obtained experimental realization results of the fractional-order Sprott's Jerk system are presented in Fig. 6. The time domain and the phase portraits' illustrations of 
Table 2 The ultimate definitions of the rearranged Sprott's Jerk systems and the nonlinear functions including in these systems for the FPAA based implementations

\begin{tabular}{|c|c|c|}
\hline The Nonlinear Functions-' $F(X(s))$ ' & The rearranged Sprott's Jerk systems & \\
\hline$F_{1}(X(s))=|1.25 X(s)|-1$ & $\begin{array}{l}X(s)=[0.1019 Y(s)+1.02 X(s)]\left[\frac{1.2628}{(s+7.8702)}\right] \\
Y(s)=[0.128 Z(s)+1.01 Y(s)]\left[\frac{1.2628}{(s+7.8702)}\right] \\
Z(s)=\left[0.118 F_{1}(X(s))+0.891 Z(s)-0.128 Y(s)\right]\left[\frac{1.2628}{(s+7.8702)}\right]\end{array}$ & (17) \\
\hline$F_{2}(X(s))=-1.2 X(s)+\operatorname{sgn}(2 X(s))$ & $\begin{array}{l}X(s)=[0.128 Y(s)+1.01 X(s)]\left[\frac{1.2628}{(s+7.8702)}\right] \\
Y(s)=[0.128 Z(s)+Y(s)]\left[\frac{1.2628}{(s+7.8702)}\right] \\
Z(s)=\left[0.112 F_{2}(X(s))+0.908 Z(s)-0.132 Y(s)\right]\left[\frac{1.2628}{(s+7.8702)}\right]\end{array}$ & (18) \\
\hline$F_{3}(X(s))=0.58\left(X(s)^{2}-1\right)$ & $\begin{array}{l}X(s)=[0.128 Y(s)+X(s)]\left[\frac{1.2628}{(s+7.8702)}\right] \\
Y(s)=[0.128 Z(s)+Y(s)]\left[\frac{1.2628}{(s+7.8702)}\right] \\
Z(s)=\left[0.256 F_{3}(X(s))+0.921 Z(s)-0.128 Y(s)\right]\left[\frac{1.2628}{(s+7.8702)}\right]\end{array}$ & (19) \\
\hline$F_{4}(X(s))=1.6 X(s)\left(0.2 X(s)^{2}-1\right)$ & $\begin{array}{l}X(s)=[0.128 Y(s)+X(s)]\left[\frac{1.2628}{(s+7.8702)}\right] \\
Y(s)=[0.128 Z(s)+Y(s)]\left[\frac{1.2628}{(s+7.8702)}\right] \\
Z(s)=\left[0.268 F_{4}(X(s))+0.924 Z(s)-0.128 Y(s)\right]\left[\frac{1.2628}{(s+7.8702)}\right]\end{array}$ & (20) \\
\hline$F_{5}(X(s))=-0.9 X(s)\left(2.128 X(s)^{2}-1\right)$ & $\begin{array}{l}X(s)=[0.128 Y(s)+1.01 X(s)]\left[\frac{1.2628}{(s+7.8702)}\right] \\
Y(s)=[0.128 Z(s)+0.99 Y(s)]\left[\frac{1.2628}{(s+7.8702)}\right] \\
Z(s)=\left[3.19 F_{5}(X(s))+0.89 Z(s)-0.128 Y(s)\right]\left[\frac{1.2628}{(s+7.8702)}\right]\end{array}$ & (21) \\
\hline$F_{6}(X(s))=-2.15[X(s)-\tanh (2 X(s))]$ & $\begin{array}{l}X(s)=[0.128 Y(s)+X(s)]\left[\frac{1.2628}{(s+7.8702)}\right] \\
Y(s)=[0.128 Z(s)+Y(s)]\left[\frac{1.2628}{(s+7.8702)}\right] \\
Z(s)=\left[0.256 F_{6}(X(s))+0.924 Z(s)-0.128 Y(s)\right]\left[\frac{1.2628}{(s+7.8702)}\right]\end{array}$ & (22) \\
\hline
\end{tabular}


the fractional-order Sprott's Jerk system including the nonlinear functions in Eqs.2-7 have been given separately in Figs.6a-f, respectively. As seen from these figures, these experimental results agree well with the numerical simulation results in Fig.1. Therefore, the proposed LPF based implementations of the fractional-order systems are achieved with the FPAA based designs, successfully.

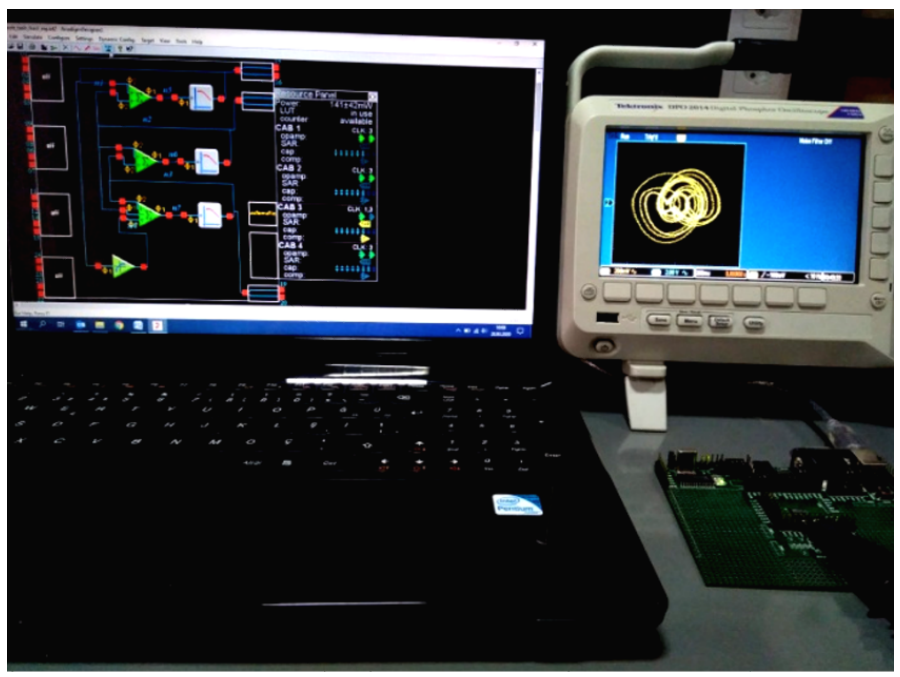

Figure 5 A photograph for the experimental setup of the FPAA based implementation of the fractional-order Sprott's Jerk system

\section{DISCUSSION AND CONCLUSION}

In this study, an effective and alternative design process has been introduced to implement the fractional-order chaotic systems with the electronic hardware. According to this procedure, the state variables of the fractional-order chaotic systems have been emulated by the modified first order low-pass filters (LPF), so the hardware usage costs and the circuit complexities have been decreased at the beginning of the hardware design process. Thus, the introduced method has been tested on the fractional-order Sprott's Jerk systems and these systems have been implemented with a programmable analog device.

In this context, firstly, the minimum fractional-orders of the state variables of the Sprott's Jerk systems have been calculated by considering the stability of the fractional-order nonlinear systems and the calculation of the minimum fractional-order has been exemplified in detail for a nonlinear function. After a common fractional order was identified for all nonlinear functions of the Sprott's Jerk system, these fractional-order systems have been simulated for $q=0.95$ by employing the G-L fractional derivative method. Thus, both the fractional derivative concepts have been handled and the results of the stability analyses have been verified by the G-L fractional derivative method. After the identification of the fractional order for the Sprott's Jerk system, the modified first order low-pass filters (LPFs), which emulate the state variables of the fractional-order chaotic systems, have been dealt with in detail. The most important arguments about this LPF structure are listed as following: i) the modified LPF structure has been characterized by depending on the changes of the fractional order and the radial frequency, ii) the desired fractional-order integrator has been built by using this LPF, iii) the adjustment of the discrete devices' values is hard to get the characteristic of the proposed LPF. On the other hand, since the identification of the "BILINEAR FILTER"

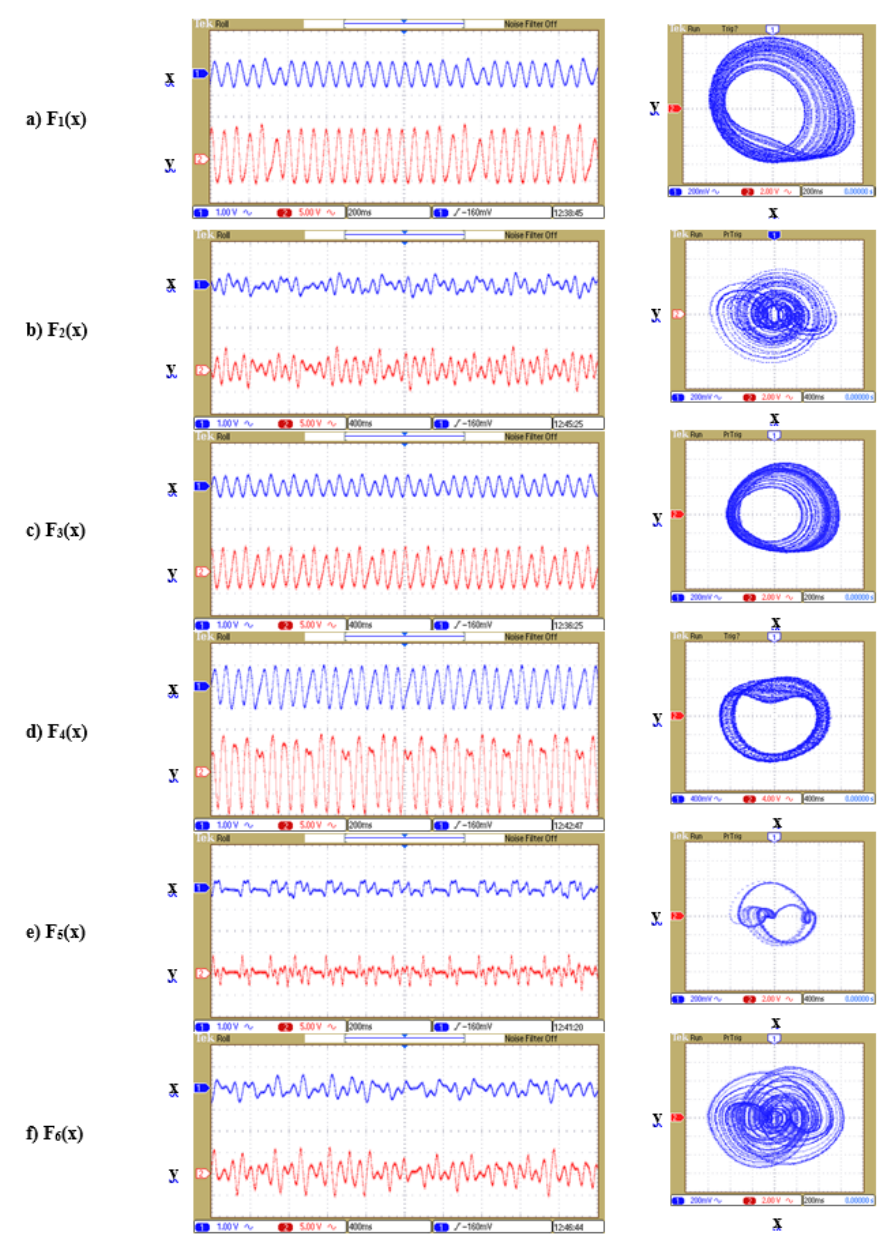

Figure 6 The time domain and the phase portraits illustrations of the fractional-order Sprott's Jerk system including the a) $F_{1}(x)$ function in Eq.2, b) $F_{2}(x)$ function in Eq.3, c) $F_{3}(x)$ function in Eq.4, d) $F_{4}(x)$ function in Eq.5, e) $F_{5}(x)$ function in Eq.6, and f) $F_{6}(x)$ function in Eq.7

CAM block in the FPAA device is very similar to the proposed LPF structure's one, this CAM block has been used as a fractionalorder integrator emulator in this study, successfully. Therefore, the fractional-order Sprott's Jerk system has been implemented by using the FPAA devices in order to prove the effectiveness of the LPF-based approximation for the fractional-order integrators. Additionally, the fractional-order Sprott's Jerk systems including the different nonlinear functions have been realized with a programmable analog device, namely FPAA. Moreover, the proposed LPF characteristic can be employed as a fractional-order integrator in the various research fields, where the desired frequency band is limited.

\section{Conflicts of interest}

The authors declare that there is no conflict of interest regarding the publication of this paper.

\section{Availability of data and material}

Not applicable. 


\section{LITERATURE CITED}

1999 Chapter 7 - numerical evaluation of fractional derivatives. In Fractional Differential Equations, edited by I. Podlubny, volume 198 of Mathematics in Science and Engineering, pp. 199-221, Elsevier.

Ahmad, W., R. El-Khazali, and A. Elwakil, 2001 Fractional-order wien-bridge oscillator. Electronics Letters 37: 1110-1112.

Ahmad, W. M. and J. C. Sprott, 2003 Chaos in fractional-order autonomous nonlinear systems. Chaos, Solitons \& Fractals 16: 339-351.

Arena, P., 2000 Nonlinear noninteger order circuits and systems: an introduction, volume 38 . World Scientific.

Atangana, A. and B. S. T. Alkahtani, 2015 Extension of the resistance, inductance, capacitance electrical circuit to fractional derivative without singular kernel. Advances in Mechanical Engineering 7: 1687814015591937.

Azar, A. T., A. G. Radwan, and S. Vaidyanathan, 2018 Mathematical Techniques of Fractional Order Systems. Elsevier.

Caponetto, R., 2010 Fractional order systems: modeling and control applications, volume 72. World Scientific.

Carlson, G. and C. Halijak, 1964 Approximation of fractional capacitors $(1 / \mathrm{s})^{\wedge}(1 / \mathrm{n})$ by a regular newton process. IEEE Transactions on Circuit Theory 11: 210-213.

Charef, A., 2006 Analogue realisation of fractional-order integrator, differentiator and fractional pi $\lambda \mathrm{d} \mu$ controller. IEE ProceedingsControl Theory and Applications 153: 714-720.

Chen, G. and T. Ueta, 2002 Chaos in circuits and systems $\sim$ world scientific.

Chen, L., W. Pan, R. Wu, K. Wang, and Y. He, 2016 Generation and circuit implementation of fractional-order multi-scroll attractors. Chaos, Solitons \& Fractals 85: 22-31.

Chen, Y. Q. and K. L. Moore, 2002 Discretization schemes for fractional-order differentiators and integrators. IEEE Transactions on Circuits and Systems I: Fundamental Theory and Applications 49: 363-367.

Deniz, F. N., A. Yüce, and N. Tan, 2019 Tuning of pi-pd controller based on standard forms for fractional order systems. Journal of Applied Nonlinear Dynamics 8: 5-21.

Elwakil, A. S., 2010 Fractional-order circuits and systems: An emerging interdisciplinary research area. IEEE Circuits and Systems Magazine 10: 40-50.

Gómez, F., J. Rosales, and M. Guía, 2013 Rlc electrical circuit of non-integer order. Open Physics 11: 1361-1365.

Gómez-Aguilar, J. F., A. Atangana, and V. F. Morales-Delgado, 2017 Electrical circuits $\mathrm{rc}$, lc, and $\mathrm{rl}$ described by atangana-baleanu fractional derivatives. International Journal of Circuit Theory and Applications 45: 1514-1533.

Gottlieb, H., 1996 Question\# 38. what is the simplest jerk function that gives chaos? American Journal of Physics 64: 525-525.

Khovanskii, A. N., 1963 The application of continued fractions and their generalizations to problems in approximation theory. Noordhoff Groningen.

Kiliç, R., 2010 A practical guide for studying Chua's circuits, volume 71. World Scientific.

Krishna, B. and K. Reddy, 2008 Active and passive realization of fractance device of order $1 / 2$. Active and passive electronic components 2008.

Krishna, B. T., 2011 Studies on fractional order differentiators and integrators: A survey. Signal processing 91: 386-426.

Matsuda, K. and H. Fujii, 1993 H (infinity) optimized waveabsorbing control-analytical and experimental results. Journal of Guidance, Control, and Dynamics 16: 1146-1153.
Maundy, B., A. Elwakil, and S. Gift, 2012 On the realization of multiphase oscillators using fractional-order allpass filters. Circuits, Systems, and Signal Processing 31: 3-17.

Oldham, K. and J. Spanier, 1974 The fractional calculus theory and applications of differentiation and integration to arbitrary order. Elsevier.

Oustaloup, A., F. Levron, B. Mathieu, and F. M. Nanot, 2000 Frequency-band complex noninteger differentiator: characterization and synthesis. IEEE Transactions on Circuits and Systems I: Fundamental Theory and Applications 47: 25-39.

Öztürk, I. and R. Kılıç, 2019 Higher dimensional baker map and its digital implementation with lsb-extension method. IEEE Transactions on Circuits and Systems I: Regular Papers 66: 4780-4792.

Petráš, I., 2011 Fractional-order nonlinear systems: modeling, analysis and simulation. Springer Science \& Business Media.

Radwan, A. G., A. S. Elwakil, and A. M. Soliman, 2008 Fractionalorder sinusoidal oscillators: design procedure and practical examples. IEEE Transactions on Circuits and Systems I: Regular Papers 55: 2051-2063.

Radwan, A. G., A. S. Elwakil, and A. M. Soliman, 2009 On the generalization of second-order filters to the fractional-order domain. Journal of Circuits, Systems, and Computers 18: 361-386.

Radwan, A. G. and K. N. Salama, 2012 Fractional-order rc and rl circuits. Circuits, Systems, and Signal Processing 31: 1901-1915.

Sacu, I. E. and M. Alci, 2019 A current mode design of fractional order universal filter. Advances in Electrical and Computer Engineering 19: 71-78.

Singh, N., U. Mehta, K. Kothari, and M. Cirrincione, 2020 Optimized fractional low and highpass filters of $(1+\alpha)$ order on fpaa. Bulletin of the Polish Academy of Sciences. Technical Sciences 68.

Sprott, J., 1997 Some simple chaotic jerk functions. American Journal of Physics 65: 537-543.

Sprott, J. C., 1994 Some simple chaotic flows. Physical review E 50: R647.

Sprott, J. C., 2000a A new class of chaotic circuit. Physics Letters A 266: $19-23$.

Sprott, J. C., 2000b Simple chaotic systems and circuits. American Journal of Physics 68: 758-763.

Stöckmann, H.-J., 1999 Quantum Chaos: An Introduction. Cambridge University Press.

Tavazoei, M. S. and M. Haeri, 2007 A necessary condition for double scroll attractor existence in fractional-order systems. Physics Letters A 367: 102-113.

Tavazoei, M. S. and M. Haeri, 2008 Chaotic attractors in incommensurate fractional order systems. Physica D: Nonlinear Phenomena 237: 2628-2637.

Tlelo-Cuautle, E., A. D. Pano-Azucena, O. Guillén-Fernández, and A. Silva-Juárez, 2020 Analog/digital implementation of fractional order chaotic circuits and applications. Springer.

Valsa, J., P. Dvorak, and M. Friedl, 2011 Network model of the cpe. Radioengineering 20: 619-626.

How to cite this article: Korkmaz, N., and Sacu, I. E. An efficient design procedure to implement the fractional-order chaotic jerk systems with the programmable analog platform. Chaos Theory and Applications, 3(2), 59-66, 2021. 